gaben nach kurzer Zeit klare dünnflüssige Lösungen, die beim Abdampfen leimartige Rückstände hinterliefsen. Von coagulirtem Albumin ist dieses Verhalten schon lange bekannt.

\title{
Ueber eine Verbindung von Silicium mit Wasserstoff;
}

\author{
von $\boldsymbol{F}$. Wöhler und $\boldsymbol{H}$. Buff.
}

(Aus d. VII. Bd. d. Abhandl. d. k. Gesellsch. d. Wissensch. zu Göıtingen.)

Das Aluminium als Bestandtheil einer galvanischen Kette zeigt, je nach Beschaffenheit der Flüssigkeit, in welche man es eintaucht, Analogieen bald mit den schwer oxydirbaren Metallen, bald wieder im auffallendsten Grade mit den metallischen Grundlagen der Alkalien, und im Allgemeinen von einer Flüssigkeit zur andern so mannichfaltige Beziehungen, dafs man aus seinem Verhalten in einem gewissen Falle auf dasjenige in einem andern Falle bisher kaum mit einiger Sicherheit Folgerungen zu ziehen vermochte. Betrachtungen dieser Art gaben uns zunächst die Veranlassung, die electrische Einwirkung des Aluminiums auf neutrale Chlorsalze einer Prüfung zu unterwerfen.

In den Lösungen des Chlornatriums, des Chlorammoniums, des Mangan- und Eisenchlorürs wird das Aluminium bei gewöhnlicher Temperatur kaum bemerkbar und selhst in der Siedhitze nur sehr wenig, obwohl unter sichtbarer Entwickelung von Wasserstoffgas angegriffen. Nach Tage langer Einwirkung bemerkt man Spuren von Thonerdehydrat in Gestalt einzeIner zarter Flocken. Da man beim Zink, Eisen und bei anderen Metallen, die sich in Säuren unter Wasserstoffent- 
wickelung auflösen, die Erfahrung gemacht hat, dafs diese directe chemische Action unter dem Einflusse des electrischen Stroms immer vermindert und oft sogar ganz unterbrochen wird, so durfte man erwarten, dafs das Aluminium, als positives Ende einer galvanischen Kette in Kochsalzlösung getaucht, nicht den geringsten directen chemischen Angriff erfahren werde. Wir waren daher überrascht, einen eingetauchten Aluminiumstab, vom Augenblicke des Schliefsens einer Kette von 8 bis $12 \mathrm{Bunsen}$ 'schen Paaren, unter starker Gasentwickelung an seiner Oberfläche sich auflösen zu sehen; und unsere Aufmerksamkeit wurde noch mehr gespannt, als einzelne der aufsteigenden Gasblasen bei dem Zerplatzen in der Luft sich von selbst entzündeten, und mit weifser Flamme, unter Erzeugung eines weilsen Rauchs verbrannten.

Dieses eigenthümliche Gas, in Glasröhren, die mit Salzwasser gefüllt waren, gesammelt, liefs sich über der Salzlösung unverändert aufbewahren. Wenn man aber eine Luflblase oder eine Blase reines Sauerstoffgas zutreten liefs, explodirte es augenblicklich unter Feuererscheinung, indem sich zugleich der ganze innere Raum mit einem weilsen Nebel erfüllte. Zuweilen konnten mehrere Sauerstoffblasen nach einander immer von Neuem Entzündung bewirken. Doch verschwand dadurch in allen Fällen nur ein kleiner Theil des Gasinhaltes. Der Rest, der sich dann nicht mehr bei der Berührung mit Sauerstoff von selbst entzündete, eudiometrisch geprüft, verhielt sich wie Wasserstoffgas.

An dem Aluminiumstab als positivem Pole hatle sich also Wasserstoffgas entwickelt, dem eine geringe Menge eines anderen, selbstentzündlichen Gases beigemengt war.

Die Leichtigkeit, womit sich dieses Gasgemenge beim Zutritt der Luft entzündete, blieb sich übrigens nicht immer gleich. Zuweilen geschah es unter heftigen Explosionen, begleitet von glänzender Lichterscheinung. Zuweilen wieder 
trat die Entzündung nicht freiwillig ein, konnte aber herbeigeführt werden, indem man die auf der Flüssigkeit schwimmende Blase mit einem heifsen Platindrahte berührte. Viele der aufsteigenden Blasen konnten selbst durch dieses Hülfsmittel nicht entzündet werden. Diese letzteren konnten folglich nur wenig oder nichts von dem selbstentzündlichen Gase enthalten haben. Wir erkannten bald, dals diese Verschiedenheiten theils von der Beschaffenheit des angewendeten Aluminiums, theils aber auch von der Erzeugungstemperatur des Gases abhängig waren. Wenn die Flüssigkeit, aus der es sich entwickelte, durch lange Dauer eines starken Stroms nach und nach erhitzt worden war, oder wenn man die Steigerung der Temperalur dadurch förderte, dals der Salzlösung nur eine kleine Aluminiumfläche dargeboten wurde, so nahm die Menge des selbstentzündlichen Gases $a b$, und endlich blieb es ganz aus. Die Abnahme dieses Gases und selbst sein gänzliches Ausbleiben war ohne merklichen Einflufs auf die Gasentwickelung im Allgemeinen. Die Erscheinung des selbstentziindlichen Gases konnte daher nur in einem untergeordneten Zusammenhange stehen mit der Art der Einwirkung des Aluminiums anf die Salzlösung. Eine weitere Bestätigung erhielt diese Folgerung durch die Erfahrung, dals die Gasentwickelung am positiven Pole, bei verschiedenen Aluminiumstücken von ungleicher Reinheit, sich so ziemlich unverändert zeigte, während die reichlichste Ausbeute an selbstentzündlichem Gase besonders von solchen Stücken erhalten wurde, die ziemlich viel Silicium enthielten.

Gestülzt auf diese Erfahrungen benutzten wir zur Darstellung des Gases in der Folge vorzugsweise ein an Silicium reiches Aluminium, wozu das aus Paris im Handel vorkommende schon hinreichend geeignet ist, da es stets eine bedeutende Menge Silicium enthält; zugleich vermieden wir während der Dauer des electrolytischen Vorgangs möglichst 
eine starke Erhitzung der Flüssigkeit. Wenn wir dadurch sicher waren, das selbstentzündliche Gas immer wieder erhalten zu können, so blieb gleichwohl das quantitative Verhältnifs desselben immer nur gering. Da wir überdiels kein Mittel fanden, dasselbe, ohne es zu zerstören, von dem in grofsem Uebermafse beigemengten Wasserstoff zu trennen, so mufsten wir uns vorläufig auf die qualitative Untersuchung beschränken, aus der indessen unzweifelhaft hervorgegangen ist, dafs dieses selbstentzündliche Gas eine Verbindung von Silicium mit Wasserstoff ist.

Wir verschafften uns zu diesem Zweck gröfsere Quantitäten, bis zu $300 \mathrm{CC}$. des Gasgemenges. Sie wurden in einer am oberen Ende durch einen Hahn verschlielsbaren Glasglocke gesammelt. Liefs man dieses Gas durch die Oeffnung des Hahns unmittelbar in die Luft austreten, so entzündete es sich gewöhnlich von selbst und verbrannte mit weilser leuchtender Flamme, indem sich an dem Rande der Ausmündung ein weifser Anflug absetzte, der sich wie Kieselerde verhielt.

Wenn eine Scheibe von weilsem Porcellan gegen die Flamme gehalten wurde, so bildeten sich daran Flecken, je nach ihrer Dicke von bräunlichgelber bis chocoladebrauner Farbe. Dieselben veränderten sich nicht in der Löthrohrflamme, waren in Wasser und Säuren unlöslich, lösten sich aber in Aetzkali unter Gasentwickelung. Diese Substanz konnte also nur aus Silicium bestehen.

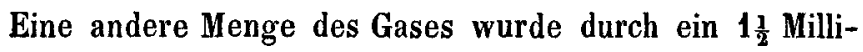
meter weites Rohr von schwer schmelzbarem Glase geleitet, in welches man zuvor mehrere schmale Streifen Platin gebracht, dann bis zum Erweichen des Glases erhitzt hatte. Die Platinfläche so wie die Glaswände bedeckten sich mit dem vorerwähnten braunen Anfluge, der auf dem Glase spiegelnd erschien und gleich dem aus der Flamme abgeschie- 
denen die characteristischen Eigenschaften des amorphen Siliciums zeigie. Das der äufseren Mündung des Glasrohrs entströmende Gas entzündete sich jetzt nicht mehr von selbst. Angezündet war seine Flamme gleichwohl heller als die des reinen Wasserstofls; auch bildete sie noch immer einen weifsen Nebel. Ein Theil des leicht entzündlichen Gases schien hiernach zurückgeblieben zu sein, obschon die gröfsere Menge augenscheinlich unter Abscheidung von Silicium zersetzt worden war. Die Gewichtszunahme des Glasrohrs betrug dessenungeachtet nur 4,5 Milligrm. auf $250 \mathrm{CC}$. des ursprünglichen Gasgemenges.

Bei einer Wiederholung dieses Versuchs wurde das aus dem erhitzten Glasrohr strömende Gas über Salzwasser in einer graduirten Glocke aufgefangen. Es schien sein anfängliches Volum nicht geändert zu haben. Sicher liefs sich darüber nicht entscheiden, weil in der Röhrenverbindung beider Glocken etwas Luft zurückgeblieben war, und weil als Verbindungsstücke Gummischläuche angewendet werden mufsten. Kleine Volumänderungen konnten daher der Wahrnehmung entgangen sein. Mit Hülfe des folgenden genaueren Verfahrens zeigte sich, dafs in der That eine geringe Volumvermehrung staltgefunden hatte.

Es wurden $183 \mathrm{CC}$. des Gases bei $23^{\circ}, 2 \mathrm{C}$. und unter $331^{\prime \prime, 1}$ Druck in einer $33^{\mathrm{mn}}$ weiten, graduirten Glasglocke gesammelt. Letztere stand in einem hohen, mit Salzwasser gefüllten $\mathrm{Cy}$ linderglase. In diese Glocke wurde von unten ein dünner Platindraht eingeschoben, dessen beide Enden um dickere, in gebogene Glasröhren geschmolzene Platinstücke gewickelt waren. Die doppelschenkelig gebogenen Röhren enthielten Quecksilber und gestatteten dadurch eine leitende Verbindung des Drahts nach Aufsen. Diese Anordnung war getroffen worden, um den dünnen, sich fast durch die ganze Länge der Glocke in doppelter Linie erstreckenden Draht mittelst 
des electrischen Stroms zum Glühen bringen zu können. Die Erreichung dieses Zwecks wurde indessen durch den bekannten abkühlenden Einfluls des Wasserstoffs über alle Erwartung erschwert, und erforderte einen Strom, dessen Stärke diejenige, wobei derselbe Draht in der Luft zum Glühen kam, um mehr als das Dreifache übertraf. Während des Glühens bedeckte sich der Draht seiner ganzen Länge nach mit Silicium. Leider wurde aber auch die Innenwand der Glasglocke theilweise mit einem dünnen Anfluge davon bedeckt, wodurch die Hoffnung, auf diesem Wege zugleich eine genauere Gewichtsbestimmung des Siliciumgehaltes zu gewinnen, getäuscht wurde. Nach dem vollständigen Erkalten des Glases ergab sich bei 210 Lufttemperatur und unter 331 ",54 Druck ein Gasvolum von $190 \mathrm{CC}$., welches sich nach mehrmals wiederholtem Erglühen des Platindrahts und Abkühlung nicht weiter änderte. Der Zutritt von Sauerstoff zu demselben bewirkte unmiltelbar keine Entzündung mehr, und als dieselbe durch erneuertes Glühen des Platindrahts herbeigeführt wurde, bildeten sich keine weilsen Nebel. Das selbstentzündliche Gas war also unter Abscheidung von Silicium vollständig zerstört worden. Die beiden gemessenen Gasvolume auf $0^{\circ}$ und $336^{\prime \prime}, 9$ Druck reducirt, gaben 165,76 und 173,61 CC. Das anfängliche Volum hatle also durch Entfernung seines Siliciumgehaltes um 7,85 CC. zugenommen. In einem andern Falle waren 70,66 CC. des Gasgemenges in $\mathbf{7 4 , 7 9}$ reinen Wasserstoff verwandelt worden.

Diese Erfahrungen sind leider unzureichend, um über die quantitative Zusammensetzung des selbstentzündlichen Gases Aufschlufs zu geben; doch dürfen wir als ausgemacht betrachten, dafs dieses Gas eine Verbindung ist von Silicium mit Wasserstoffgas, dessen Volum dabei eine Verdichtung erfahren hat. 
Das Silicium-Wasserstoffgas ist in reinem, luftfreiem Wasser, gleich wie im Salzwasser unlöslich. Verdünnte Schwefelsäure und Salzsäure lassen dasselbe unverändert. Mit Aetzkalilösung geschüttelt wird es schon bei gewöhnlicher Temperatur zersetzt, und zwar unter Vermehrung des Gasvolums. Mit Chlor entzündet es sich ähnlich und selbst noch leichter als mit Sauerstoff.

Aus den Lösungen des Chlorkaliums, Chlorammoniums, des Eisen- und Manganchlorürs und selbst des Chloraluminiums entwickelt das Aluminium als positiver Pol, ähnlich wie aus Salzwasser, ein Gemenge von Wasserstoffgas mit wenig Silicium-Wasserstoffgas. Auch in Salzsäure, so weit verdünnt, dals sie das Aluminium für sich nur wenig angreift, entstand unter Mitwirkung des Stroms sogleich eine starke Entwickelung von Wasserstoffgas mit einzelnen Blasen von Siliciumwasserstoffgas das sich an der Luft entzündete.

Wir haben eine grolse Zahl von Versuchen gemacht, die Bildung des Siliciumwasserstoffs auf rein chemischem Wege in die Gewalt zu bekommen, ohne aber bis jetzt den $Z$ weck zu erreichen. Nur noch auf eine Art haben wir seine Bildung, wiewohl nur in kleiner Menge, beobachtet, nämlich bei der Auflösung von siliciumhaltigem Aluminium in verdünnter Chlorwasserstoffsäure. Wird das sich entwickeInde und durch Chlorcalcium getrocknete Wasserstoffgas angezündet, so brennt es mit leuchtenderer Flamme als reines Wasserstoffgas, und hält man gegen dieselbe eine kalte Glasfläche, so bildet sich darauf ein weifser Hauch von Kieselerde und selbst zuweilen ein bräunlicher Hauch von Silicium. Treibt man das getrocknete Gas durch ein an einer Stelle glühendes enges Glasrohr, so bildet sich hier ein sehr deutlicher brauner Spiegel von Silicium. Nie aber erhielten wir auf diese Weise ein an Siliciumwasserstoff so reiches Gas, 
dafs es sich von selbst entzündete, selbst wenn wir ein Aluminium anwandten, das durch Schmelzen mit Wasserglas und Kryolith *) mit Silicium übersättigt war. Wir vermuthen, dals das den electrolytischen Vorgang begleitende Siliciumwasserstoffgas einen gleichen Ursprung hat, dals nämlich in beiden Fällen Wasserstoff im Entstehungszustande mit dem im Aluminium enthaltenen Silicium in Berïhrung kommt. Jedoch scheint nur die mit dem Aluminium chemisch verbundene kleine Menge Silicium, und nicht das blofs Eingemengte die Verbindung mit dem Wasserstoff eingehen zu können, denn die bei weitem gröfsere Menge des Siliciums fällt während der Auflösung des Aluminiums davon ab, theils in Gestalt krystallinischer Blätlchen, theils als feines schwarzes Pulver.

Wenn das Aluminium, sei es mit rein metallischer Oberfläche oder bereits schon mit Silicium überdeckt, als negativer Pol einer galvanischen Kette in eine Salzlösung getaucht wird, so bildet sich keine Spur von selbstentzündlichem Gase, das Aluminium wird nicht angegriffen und verliert nichts von seinem Gewichte.

Die sonderbare Eigenschaft des Aluminiums, in den Lösungen vieler Chlorsalze, in welchen es für sich unauflöslich ist, sowohl als negativer wie als positiver Pol einer galvanischen Kette Wasserstoffgas auszuscheiden, eine Eigenschaft, die, soweit uns bekannt, ohne Analogie ist, kann nur die Folge sein eines vom rein electrolytischen Vorgange in secundärer Abhängigkeit stehenden chemischen Processes. Darauf deutet zunächst der Umstand hin, dals die Gasmengen an beiden Polen in keinem einfachen und ganz unveränderlichen Verhältnisse zu stehen scheinen. In der folgenden Tafel sind einige der von uns ausgeführten Messungen zusammengestellt. Die bezeichneten Ablenkungen beziehen

*) Vergl. diese Annalen CII, 382. 
sich auf die Nadel einer Tangentenbussole, haben jedoch nur eine approximative Geltung, da man kein Gewicht darauf legte; die Stromstärke im Laufe eines Versuchs absolut unveränderlich zu erhalten,

$\begin{array}{cccc}\begin{array}{c}\text { Ablenkung der Nadel } \\ \text { in Graden }\end{array} & \begin{array}{c}\text { Wasserstoffgas in CC. am } \\ - \text { Pole } \\ 10^{\circ}, 9\end{array} & \begin{array}{c}\text { Verhältnifs beider } \\ \text { Gasmengen } \\ \text { wie } 100 \text { zu }\end{array} \\ 20^{\circ}, 7 & 27,4 & 5,5 & 23,50 \\ 29^{\circ}, 0 & 39,5 & 6,0 & 22,14 \\ 34^{\circ}, 0 & 31,5 & 9,25 & 20,88 \\ 35^{\circ}, 0 & 695,6 & 165,8 & 30,95\left(^{*}\right) \\ 46^{\circ}, 0 & 52,5 & 11,5 & 23,83 \\ 48^{\circ}, 0 & 52,0 & 12,0 & 21,90 \\ & & & 23,08 .\end{array}$

Mit einziger Ausnahme des vierten mit (*) bezeichneten Versuchs enthielt das Gas des positiven Pols stets SiliciumWasserstoff. Bei diesem Versuche war nur cine kleine Aluminiumfläche dem Strom ausgesetzt worden, die Zersetzung ging unter starker Erwärmung vor sich, und in Folge der Erhitzung der Flüssigkeit dauerte die Gasentwickelung auch nach Unterbrechung des Stroms mil abnehmender Stärke noch einige Zeit fort. In allen übrigen Fällen hälten durch Ausscheidung des Siliciums die in der vierten Spalte enthaltenen Zahlen noch etwas zunehmen müssen. So erhielt man im fünften Versuche aus 165,8 CC. Gas durch Abscheidung des Siliciums 173,6 CC., was fast genau $\frac{1}{4}$ von dem am negativen Pole gesammelten Gase ausmacht. Die Messungen waren mit grofser Sorgfalt ausgeführt und auf $0^{\circ}$ und 336",9 reducirt. Doch mag das Verhältnifs 4:1 nur ein zufälliges sein. Jedenfalls sehen wir bis jetzt keinen nothwendigen Grund dafür ein.

Die Gewichtsmenge des aufgelösten Aluminiums ist mehrmals mit der Wirksamkeit des electrolytischen Vorgangs, als deren Mals das Volum des am negativen Pole entwickelten 
Wasserstoffs dienen konnte, verglichen worden. So wurde erhalten :

Wasserstoffgas bei $0^{\circ}$ und unter $336^{\prime \prime \prime}, 9$ Druck

$\begin{array}{rr}\text { CC. } & \text { Milligrm } \\ 51,9 & 4,65 \\ 222,2 & 19,90 \\ 240,3 & 21,50\end{array}$

Gewichtsverlust des Aluminiumdrahts in Milligrm. gefunden berechnet $53,5 \quad 42,5$ $247,8 \quad 181,8$ $257,6 \quad 196,6$.

Der berechnete Gewichtsverlust entspricht der Annahme, dafs 3 Aequivalente des am Aluminium sich abscheidenden Chlors sich mit 2 Aeq. Aluminium zu Aluminiumchlorid verbanden. Die wirkliche Gewichtsabnahme betrug aber reichlich um $\frac{1}{4}$ mehr. Nur ein kleiner Theil dieses Unterschiedes kann auf Rechnung des während der Auflösung des Aluminiums sich gleichzeitig losreilsenden Siliciums gebracht werden. Die beiden ersten Versuche waren mit einem Drahte ausgeführt worden, der nur Spuren von Silicium enthielt. Der bei dem dritten Versuche benutzte Draht enthielt 6,25 pC. Silicium. Im Gewichtsverluste konnten hiernach ungefähr 16 Milligrm. Silicium eingeschlossen sein. Damit stimmte die directe Bestimmung gul überein. Man hatte nämlich die electrolytische Auflösung des Drahts in einer besonderen porösen Zelle (gebildet aus einem weiten Glasrohr, dessen untere Oeffnung mit Blase umbunden war) vor sich gehen lassen, wodurch es leicht wurde, die Abfälle zu sammeln, nach sorgfältigem Auswaschen unter der Luftpumpe zu trocknen und zu wägen. Man fand 13 Milligrm. Diese Masse enthielt kein Aluminium mehr, denn Salzsäure löste nichts davon auf. Ausglühen bewirkte keine Aenderung des Gewichtes. Da höchstens einige Milligramme Silicium in Verbindung mit Wasserstoff fortgegangen scin konnten, so nahmen wir 16 Milligrm. als den Siliciumgehalt des Gewichtsverlustes der Aluminiummasse. Das wirklich aufgelöste Aluminium betrug demnach $25 \%, 6-16=241,6$, während als 
Aluminiumchlorid nur 196,6 hatte aufgenommen werden können. Der Unterschied von 45 Gewichtstheilen, d. h. nahe $\frac{1}{4}$ der berechneten Menge konnte sich nicht mit Chlor verbunden haben, und mufste folglich in Form von Thonerde in die Flüssigkeit übergegangen sein.

Hierdurch nun erklärt sich die Bildung von Wasserstoffgas am positiven Pole, deren Menge ebenfalls beiläufig von dem electrolytisch abgesetzten Wasserstoff ausmachte.

In den unlöslichen Abfällen des positiven Pols fand sich keine Thonerde; dieselbe mufste sich folglich mit dem gleichzeitig entstandenen Chloraluminium zu einem im Wasser löslichen basischen Aluminiumchlorid vereinigt haben. In der That läist sich die Existenz einer solchen Verbindung leicht darthun.

Das Aluminium wird, wie bereits bemerkt wurde, in reiner Salzlösung äufserst wenig, jedoch unter Abscheidung von Thonerdehydrat angegriffen. Nimmt man anstatt der reinen Salzlösung eine solche, in welcher durch den electrischen Strom in einer besonderen, den positiven Pol umschliefsenden porösen Zelle sich Chloraluminium gebildet hatte, worin also in keinem Falle freie Säure, aber auch kein freies Alkali enthalten sein konnte, so wird ein Aluminiumdraht, zumal in der Hitze, merklich mehr als in reiner Salzlösung angegriffen. Gleichwohl entsteht kein Niederschlag von Hydrat. Dagegen verschwindet das in reiner Salzlösung erzengte Hydrat, wenn chloraluminiumhaltige Salzlösung zugesetzt und damit geschüttelt, oder wenn das Gemische erwärmt wird.

Ebenso wird der in einer Lösung von sublimirtem Chloraluminium durch Zusatz einiger Tropfen Ammoniak gebildete Niederschlag durch Schütteln, oder rascher durch Erwärmen in Menge wieder aufgenommen und Aluminium wird in einer 
Lösung dieses Salzes unter Wasserstoffgas-Entwickelung in Menge aufgelöst.

Wenn man die Electrolyse des Salzwassers in zweien durch eine poröse Wand getrennten Zellen vor sich gehen läfst, dann die Flüssigkeiten beider Zellen vermischt, so wird das am positiven Pole dargestellte basische Chloraluminium durch das am negativen Pole entstandene Aetznatron vollständig ausgefällt.

Das Auftreten von Wasserstoffgas am electropositiven Aluminiumdraht in Kochsalzlösungen, liels uns anfänglich vermuthen, dafs unter Vermittelung des electrischen Stroms ein Aluminium-Chlorür gebildet werde, das dann durch die Berührung mit Wasser unter Wasserstoffentwickelung sich theilweise in Thonerde umwandle. Wir mufsten jedoch diese Annahme wieder fallen lassen, indem es uns auf keinem anderen Wege gelungen ist, dieses hypothetische Chlorür, sei es für sich oder in Verbindung mit anderen Körpern, darzustellen. Wir erhielten es z. B. nicht, als wir durch ein mit Aluminiumstücken gefülltes, bis kaum zum Glühen erhitztes Glasrohr Chlorwasserstoffgas leiteten, welches dabei sehr leicht und vollständig zersetzt wurde, aber unter Bildung des gewöhnlichen Chloraluminiums, $\mathrm{Al}^{2} \mathrm{Gl}^{3}$.

Es scheint somit, dafs die leichte Auflöslichkeit des basischen Chloraluminiums die einzige Ursache ist, warum unter dem Einflusse des Stroms das Aluminium als positiver Pol einer Kette in gröfserer Menge aufgelöst wird, als sich mit dem an seiner Oberfläche electrisch abgesetzten Chlor direct verbinden kann. 